\title{
$\beta$ Cells led astray by transcription factors and the company they keep
}

\author{
Peter Thompson and Anil Bhushan
}

Diabetes Center, UCSF, San Francisco, California, USA.

\begin{abstract}
Pancreatic $\beta$ cells have one of the highest protein secretion burdens in the body, as these cells must synthesize and secrete insulin in proportion to postprandial rises in blood glucose. Remarkably, it is now becoming clear that adult $\beta$ cells retain plasticity and can dedifferentiate into embryonic fates or adopt alternate islet endocrine cell identities. This property is especially important, because changes in cell fate alter $\beta$ cell function and could form the basis for defects in insulin secretion that occur early in the pathogenesis of the most prevalent form of $\beta$ cell dysfunction, type 2 diabetes. In this issue, three different studies provide complementary perspectives on how the transcription factors NK2 homeobox 2 (NKX2.2), paired box 6 (PAX6), and LIM domain-binding protein 1 (LDB1) serve to maintain mature adult $\beta$ cell identity, revealing clues as to how adult $\beta$ cells can partially dedifferentiate or become reprogrammed into other islet endocrine cells.
\end{abstract}

crine cell fates as a consequence of various stressors $(2,4,17)$. Importantly, these studies probe the different relationships of NKX2.2, PAX6, and the LIM domainbinding protein 1-islet 1 (LDB1-ISL1) complex to the chromatin modification machinery in adult $\beta$ cells and show how the expression and function of these TFs are sensitive to environmental and metabolic changes associated with T2D.

TFs: maintenance of $\beta$ cell identity on different levels $\beta$ cell-specific ablation of $N k x 2.2, \operatorname{Pax} 6$, or $L d b 1$ in adult animals resulted in a similar suite of metabolic and physiologic defects in $\beta$ cell function, including loss of insulin content, defects in insulin secretion, and glucose intolerance, with or without accompanying fasting or fed hyperglycemia (8-10). However, the underlying cause of these related phenotypes was found to be quite different in each case. Dominguez et al. showed that $N k x 2.2-\mathrm{KO} \beta$ cells become partially transdifferentiated into $\alpha$ - and $\delta$-like cells (8). This phenotype, along with the ensuing hyperglycemia, is reminiscent of the $\beta$ cell transdifferentiation phenotype that is observed upon deletion of $P d x 1$ or Nkx6.1 in adult $\beta$ cells, respectively (12, 14). NKX2.2 functions primarily as an activator of $\beta$ cell genes (including TFs and glucose metabolic genes) and as a repressor of metabolically disallowed genes and genes expressed by other islet endocrine cell types (8). Similarly, Swisa and colleagues demonstrated that adult $\beta$ cells lacking PAX6 also become partially transdifferentiated by acquiring ghrelin expression (9). PAX6 was found to function as both an activator and repressor, co-occupying enhancers bound by the $\beta$ cell-specific TFs PDX1, NKX6.1, MAFA, FOXA2, and NEUROD1. Additionally, PAX6 also indirectly maintained repression of the undifferentiated endocrine progenitor program through regulation of Foxa2 (9). Ediger et al. determined that 

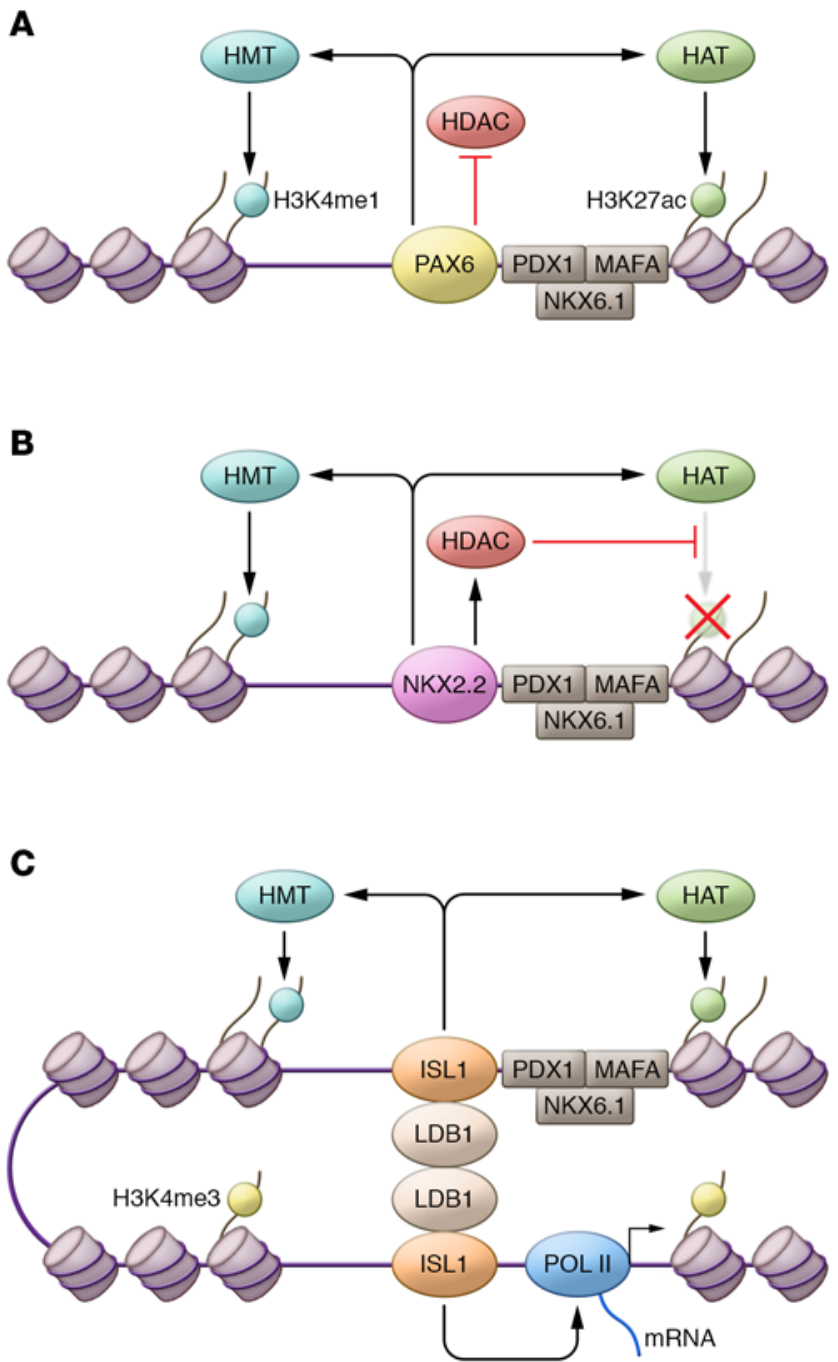

LDB1, in concert with its DNA-binding interactor ISL1, is required to maintain the active state of enhancers, thereby supporting the function of the $\beta$ cellspecific TFs PDX1, NKX6.1, and FOXA2 (10). It should be noted, however, that the metabolic and physiologic consequences of the adult $\beta$ cell-KO phenotype for $L d b 1$ and Isl1 were less severe than the consequences observed in $\beta$ cells lacking the TFs that functionally interact with LDB1ISL1, suggesting that the $\beta$ cell TFs may perform some of their gene-regulatory functions independently of LDB1-ISL1. Therefore, while different in their adult $\beta$ cell-KO phenotypes, NKX2.2 and PAX6 may have similar functional roles in providing activator and repressor activities at enhancers and a small subset of promoters. In contrast, LDB1-ISL1 serve as chromatin structural factors to maintain $\beta$ cell enhancer activity.
TFs and chromatin modifiers: keeping company in adult $\beta$ cells During pancreas development and endocrine lineage specification, a sequential cascade of TFs is known to control the ordered differentiation process to a pan-endocrine fate that ultimately culminates in the production of the six different islet endocrine cell types, including $\beta$ cells. At each stage, epigenetic mechanisms, including DNA methylation and histone modifications, provide so-called "memory" for particular gene functional states (e.g., active, repressed, poised). Thus, while TFs may establish a cell's identity, epigenetic mechanisms are generally thought to maintain this identity, such that the perpetual activity of the pioneering TFs may not always be necessary. Why, then, are the same developmental TFs necessary to maintain particular promoter/enhancer activity states in fully matured adult $\beta$ cells? Some clues
Figure 1. Roles of the islet cell TFs PAX6, NKX2.2, and LDB1-ISL1 in the maintenance of adult $\boldsymbol{\beta}$ cell identity. (A) An example of how PAX6 might regulate the activity of a $\beta$ cell enhancer. PAX6 binds to its DNA sequence with a combination of different $\beta$ cell-specific TFs, represented by PDX1, NKX6.1, and MAFA. Alongside the $\beta$ cell TFs, PAX6 could modulate the activity status of the enhancer by promoting recruitment of histone methyltransferases (HMTs) that deposit H3K4me1 and histone acetyltransferases (HATs) required for $\mathrm{H} 3 \mathrm{~K} 27 \mathrm{ac}$, while inhibiting recruitment of histone deacetylases (HDACs). (B) In a scenario similar to that depicted in $\mathbf{A}, N K X 2.2$ could regulate the activity state of a poised enhancer by binding to its sequence along with $\beta$ cell-specific TFs and recruiting HMTs to maintain H3K4me1 and HDACs to maintain deacetylation of H3K27. (C) LDB1-ISL1 multimeric complexes bind sequences in the enhancer along with $\beta$ cell TFs to form looped chromatin structures, which may be necessary for high-level enhancer activity toward active (H3K4me3-marked) target promoters. Whether the looping structure is necessary for the active enhancer marks H3K4me1 and $\mathrm{H} 3 \mathrm{~K} 27 \mathrm{ac}$, or occurs as a consequence of these marks, remains to be determined.

toward an answer to this question may lie in the relationship between TFs and chromatin modifiers in terminally differentiated cells, such as $\beta$ cells, as compared with other more proliferative, nonsecretory cell types. Indeed, the $\beta$ cell-specific deletion of TFs or chromatin modifiers does not result in $\beta$ cells adopting fates outside the endocrine lineage $(8-10,12,14,15,17,18)$, suggesting that plasticity is limited to fates within the endocrine lineage and that both TFs and chromatin modifiers maintain the mature endocrine cell fate. Rather than dictating an establishment versus maintenance paradigm, TFs and chromatin modifiers may cooperate on an ongoing basis to maintain appropriate endocrine cell phenotypes (Figure 1, A and B). This mechanism may lend itself to the ability to rapidly adapt to changes in homeostasis at the transcriptional level, as is necessary for professional, nondividing secretory 
cells like the $\beta$ cells that are wired to modulate output on the basis of environmental signals. Notably, both epigenetic "writers" (e.g., histone methyltransferases and acetyltransferases and DNA methyltransferases) and "erasers" (histone demethylases, deacetylases, and ten-eleven translocation [TET] proteins) are expressed in adult mouse and human $\beta$ cells; therefore, a balance between the activities of these chromatin modifiers must be maintained at active versus repressed loci throughout the genome.

TFs can activate or repress gene expression via a wide variety of direct and indirect mechanisms (19) including: regulation of gene expression of other TFs; differences in binding partners and chromatin modifiers of these TFs; interactions with noncoding RNAs; alternatively spliced TF isoforms (which was suggested for PAX6; ref. 9); and decoy/blockade of other TFs. Thus, it is not surprising to find that NKX2.2 and PAX6 can serve as both activators and repressors of different genes in adult $\beta$ cells (Figure 1, A and B). A further layer of complexity for deciphering the functions of NKX2.2 and PAX6 in the $\beta$ cell transcriptome is evidenced by the finding that these TFs also exert regulatory effects on the activity and/or expression of other $\beta$ cell-specific TFs, including PDX1, NKX6.1, NEUROD1, MAFA, and FOXA2 $(8,9)$. The sequence context and, thus, the presence of other TFs bound to the same enhancer are likely to be major factors in determining the functional consequences of PAX6 and NKX2.2 binding (Figure 1, A and $\mathrm{B})$. These context-dependent effects were evidenced by the ability of PAX6 to activate or repress luciferase reporter constructs in Min6 cells in the same way these sequences are regulated in vivo (9). The regulatory effect of PAX6 on different enhancer constructs in Min6 cells also correlated with the degree of the histone mark $\mathrm{H} 3 \mathrm{~K} 27 \mathrm{ac}$ at each region, as determined from ChIP-sequencing (ChIP-seq) data sets on these cells (9), thereby affirming the interplay between TF binding and chromatin modification status (Figure 1, A and B). Similarly, in a previous study, NKX2.2 was found to bind and repress the aristalessrelated homeobox (Arx) promoter in $\beta$ cells by recruitment of DNA methyltransferase 3a (DNMT3a) and histone deacetylase 1 (HDAC1) complexes, and reporter assays showed that NKX2.2 directly represses the Arx promoter sequence (20). Conversely, whether chromatin modifications themselves feed back on the functions of TFs in $\beta$ cells remains to be determined. Thus, PAX6 and NKX2.2 are examples of islet cell TFs that modulate the activity status of enhancers and promoters as the result of sequence context and combinatorial interactions with other TFs and chromatin modifiers (Figure 1, A and B), as has been recently demonstrated for other groups of TFs (21). LDB1 and ISL1 function as chromatin structural/looping factors for $\mathrm{TF}$ complexes at active $\beta$ cell enhancers (Figure $1 \mathrm{C}$ ). Active enhancers are also known to produce short noncoding transcripts known as enhancer RNAs (eRNAs), and this looping might be required for eRNA production (22). Enhancer structural looping is necessary to enforce the fully differentiated $\beta$ cell transcriptional program, since $\mathrm{KO}$ of $L d b 1$ or Isl 1 in adult $\beta$ cells led to dedifferentiation into $\mathrm{Ngn}^{+}$endocrine progenitors (10). In this case, LDB1 and ISL1 are likely to be recruited with the $\beta$ cell-specific TFs themselves (ref. 10 and Figure 1C) and provide an additional layer of epigenetic information in the form of chromatin looping to sustain enhancer activity. Whether these LDB1-ISL1 looped domains are needed at all active $\beta$ cell enhancers, or just at those promoting high-level gene expression, remains to be determined. Future investigations could use chromosome conformation capture carbon copy-based (5C-based) approaches to identify genes controlled by LDB1ISL1-bound enhancers in $\beta$ cells. Moreover, recent work in hematopoietic and neurodevelopmental models has demonstrated that LDB1-enforced chromatin looping at enhancers can dictate a variety of functional states, including POL II-paused repression and developmentally controlled transcriptional activation $(23,24)$. Therefore, it will be of interest to investigate how LDB1-ISL1 complexes, which play general roles in enhancer organization in multiple lineages, orchestrate enhancer-promoter relationships in adult $\beta$ cells.

\section{TF sensitivity to stress underlies $\beta$ cell fragility}

The emerging view from recent studies suggests that $\beta$ cell TFs, including PDX1,
MAFA, FOXO1, and NKX6.1, are vulnerable to dysregulation in T2D due to metabolic stressors, such as chronic hyperglycemia and oxidative damage $(1,3,6)$. These observations dovetail nicely with the intrinsic fragility of mature $\beta$ cells when challenged with various stressors $(4,16)$ and suggest that the sensitivity of these core $\beta$ cell TFs to stress is an explanation for $\beta$ cell fragility. The studies on PAX6 and LDB1 indicate that they too fall into this category. Indeed, PAX6 mRNA and protein levels were downregulated in hyperglycemic insulin receptor antagonist-injected mice and in leptin receptor-deficient $(d b / d b)$ mice in a manner that correlated with the age-dependent severity of hyperglycemia (9). Similarly, LDB1 mRNA and protein levels were also downregulated in $d b / d b$ mice starting at 8 weeks of age (10). Whether NKX2.2 is similarly disrupted in $d b / d b$ mice or in human T2D awaits further inquiry. NKX2.2, PAX6, and LDB1 seem to play similar roles in human $\beta$ cells, as evidenced from the ChIPseq analyses of human islets (8-10), and NKX2.2 and PAX6 mutations have been found to cause neonatal diabetes in humans $(25,26)$, underscoring the functional importance of these TFs for human $\beta$ cell formation and function. In addition, it was suggested that the enhancer state maintained by LDB1-ISL1 becomes progressively compromised with age and in T2D individuals (10). Whether the observed changes in expression and function of these TFs in the face of metabolic stressors, aging, and T2D cause the functional decline of $\beta$ cells, or are merely a consequence of the pathogenesis, requires further investigation. Reversal of $\beta$ cell oxidative stress by crossing $d b / d b$ mice with glutathione peroxidase 1 (Gpx1) transgenic mice led to restoration of the appropriate expression and localization of $\beta$ cell TFs (6). In addition, upon dedifferentiation in other contexts, $\beta$ cells were also found to redifferentiate appropriately upon removal of metabolic stress (2), suggesting that this may be the case. Therefore, therapeutic approaches aimed at restoring islet cell TF expression and/or localization by mitigating stress could improve $\beta$ cell function and prevent deterioration of functional $\beta$ cell mass in T2D.

Address correspondence to: Anil Bhushan or Peter Thompson, Diabetes Center, UCSF, 513 Parnassas Avenue, San 
Francisco, California 94134, USA. Phone: 310.206.5750 (A. Bhushan), 415.691.1484 (P. Thompson); E-mail: anil.bhushan@ ucsf.edu (A. Bhushan), peter.thompson@ ucsf.edu (P. Thompson).

1. Talchai C, Xuan S, Lin HV, Sussel L, Accili D. Pancreatic $\beta$ cell dedifferentiation as a mechanism of diabetic $\beta$ cell failure. Cell. 2012;150(6):1223-1234.

2. Wang Z, York NW, Nichols CG, Remedi MS. Pancreatic beta-cell dedifferentiation in diabetes and redifferentiation following insulin therapy. Cell Metab. 2014;19(5):872-882.

3. Spijker HS, et al. Loss of $\beta$-cell identity occurs in type 2 diabetes and is associated with islet amyloid deposits. Diabetes. 2015;64(8):2928-2938.

4. Cigliola V, Thorel F, Chera S, Herrera PL. Stress-induced adaptive islet cell identity changes. Diabetes Obes Metab. 2016;18(suppl 1):87-96.

5 . Remedi MS, Emfinger C. Pancreatic $\beta$-cell identity in diabetes. Diabetes Obes Metab. 2016;18(suppl 1):110-116.

6. Guo S, et al. Inactivation of specific $\beta$ cell transcription factors in type 2 diabetes. J Clin Invest. 2013;123(8):3305-3316.

7. Brereton MF, Rohm M, Ashcroft FM. $\beta$-Cell dysfunction in diabetes: a crisis of identity? Diabetes Obes Metab. 2016;18(suppl 1):102-109.

8. Domínguez Gutiérrez G, et al. Pancreatic $\beta$ cell identity requires continual repression of non- $\beta$ cell programs. JClin Invest. 2017;127(1):244-259.
9. Swisa A, et al. PAX6 maintains $\beta$ cell identity by repressing genes of alternative islet cell types. JClin Invest. 2017;127(1):230-243.

10. Ediger BN, et al. LIM domain-binding 1 maintains the terminally differentiated state of pancreatic $\beta$ cells. J Clin Invest. 2017;127(1):215-229.

11. Piccand J, et al. Rfx6 maintains the functional identity of adult pancreatic $\beta$ cells. Cell Rep. 2014;9(6):2219-2232.

12. Schaffer AE, et al. Nkx6.1 controls a gene regulatory network required for establishing and maintaining pancreatic beta cell identity. PLoS Genet. 2013;9(1):e1003274.

13. Gu C, et al. Pancreatic $\beta$ cells require NeuroD to achieve and maintain functional maturity. Cell Metab. 2010;11(4):298-310.

14. Gao T, et al. Pdx1 maintains $\beta$ cell identity and function by repressing an $\alpha$ cell program. Cell Metab. 2014;19(2):259-271.

15. Nishimura W, Takahashi S, Yasuda K. MafA is critical for maintenance of the mature $\beta$ cell phenotype in mice. Diabetologia. 2014;58(3):566-574.

16. Dooley J, et al. Genetic predisposition for $\beta$ cell fragility underlies type 1 and type 2 diabetes. Nat Genet. 2016;48(5):519-527.

17. Bramswig NC, et al. Epigenomic plasticity enables human pancreatic $\alpha$ to $\beta$ cell reprogramming. J Clin Invest. 2013;123(3):1275-1284.

18. Dhawan S, Georgia S, Tschen SI, Fan G, Bhushan A. Pancreatic $\beta$ cell identity is maintained by DNA methylation-mediated repression of Arx. Dev Cell. 2011;20(4):419-429.
19. Spitz F, Furlong EE. Transcription factors: from enhancer binding to developmental control. Nat Rev Genet. 2012;13(9):613-626.

20. Papizan JB, et al. Nkx2.2 repressor complex regulates islet beta-cell specification and prevents $\alpha$-to- $\beta$-cell reprogramming. Genes Dev. 2011;25(21):2291-2305.

21. Stampfel G, Kazmar T, Frank O, Wienerroither S, Reiter F, Stark A. Transcriptional regulators form diverse groups with context-dependent regulatory functions. Nature. 2015;528(7580):147-151.

22. Kim TK, Hemberg M, Gray JM. Enhancer RNAs: a class of long noncoding RNAs synthesized at enhancers. Cold Spring Harb Perspect Biol. 2015;7(1):a018622.

23. Krivega I, Dale RK, Dean A. Role of LDB1 in the transition from chromatin looping to transcription activation. Genes Dev. 2014; 28(12):1278-1290.

24. Zhang F, et al. Enhancer-bound LDB1 regulates a corticotrope promoter-pausing repression program. Proc Natl Acad Sci U S A. 2015;112(5):1380-1385

25. Flanagan SE, et al. Analysis of transcription factors key for mouse pancreatic development establishes NKX2-2 and MNX1 mutations as causes of neonatal diabetes in man. Cell Metab. 2014;19(1):146-154.

26. Solomon BD, et al. Compound heterozygosity for mutations in PAX6 in a patient with complex brain anomaly, neonatal diabetes mellitus, and microophthalmia. Am J Med Genet. 2009;149A(11):2543-2546 